\title{
FSY1, a horizontally transferred gene in the Saccharomyces cerevisiae EC1118 wine yeast strain, encodes a high-affinity fructose $/ \mathrm{H}^{+}$ symporter
}

Correspondence
Virginie Galeote
galeote@supagro.inra.fr

Received 19 May 2010

Revised 9 July 2010

Accepted 6 August 2010

\author{
Virginie Galeote, ${ }^{1}$ Maïté Novo, ${ }^{1}$ Madalena Salema-Oom, ${ }^{2,3}$ \\ Christian Brion, ${ }^{1}$ Elisabete Valério, ${ }^{2}$ Paula Gonçalves ${ }^{2}$ and Sylvie Dequin ${ }^{1}$ \\ ${ }^{1}$ INRA, UMR1083, Sciences Pour l'Oenologie, 2 Place Viala, F-34060 Montpellier, France \\ ${ }^{2}$ Centro de Recursos Microbiológicos (CREM), Departamento de Ciências da Vida, Faculdade de \\ Ciências e Tecnologia, Universidade Nova de Lisboa, 2829-516 Caparica, Portugal \\ ${ }^{3}$ Centro de Investigação Interdisciplinar Egas Moniz (CiiEM), Instituto Superior de Ciências da \\ Saúde Egas Moniz, 2829-511 Caparica, Portugal
}

\begin{abstract}
Transport of glucose and fructose in the yeast Saccharomyces cerevisiae plays a crucial role in controlling the rate of wine fermentation. In S. cerevisiae, hexoses are transported by facilitated diffusion via hexose carriers $(\mathrm{Hxt})$, which prefer glucose to fructose. However, utilization of fructose by wine yeast is critically important at the end of fermentation. Here, we report the characterization of a fructose transporter recently identified by sequencing the genome of the commercial wine yeast strain EC1118 and found in many other wine yeasts. This transporter is designated Fsy1 $\mathrm{p}$ because of its homology with the Saccharomyces pastorianus fructose $/ \mathrm{H}^{+}$ symporter Fsy1p. A strain obtained by transformation of the V5 hxt1-7D mutant with FSY1 grew well on fructose, but to a much lesser extent on glucose as the sole carbon source. Sugar uptake and symport experiments showed that $F S Y 1$ encodes a proton-coupled symporter with high affinity for fructose $\left(K_{\mathrm{m}} 0.24 \pm 0.04 \mathrm{mM}\right)$. Using real-time RT-PCR, we also investigated the expression pattern of FSY1 in EC1118 growing on various carbon sources. FSY1 was repressed by high concentrations of glucose or fructose and was highly expressed on ethanol as the sole carbon source. The characteristics of this transporter indicate that its acquisition could confer a significant advantage to $S$. cerevisiae during the wine fermentation process. This transporter is a good example of acquisition of a new function in yeast by horizontal gene transfer.
\end{abstract}

\section{INTRODUCTION}

The ability of the yeast Saccharomyces cerevisiae to degrade sugars into ethanol and carbon dioxide has been used by humans for several millennia for the fermentation of wine and beer, and in baking. Indeed, grape must contains large amounts of glucose and fructose, up to $300 \mathrm{~g} \mathrm{l}^{-1}$, in equimolar quantities. S. cerevisiae prefers glucose over fructose, and glucose is hence the first sugar to be depleted from the medium during fermentation (Berthels et al., 2004; Gafner \& Schütz, 1996). A high fructose/glucose ratio may therefore cause sluggish and stuck fermentations with high levels of fructose remaining in the finished wines, which is a major problem in the wine industry (Bisson, 1999; Gafner \& Schütz, 1996).

The first essential step in the utilization of hexose sugars is their uptake by yeast cells. In yeast, there are two types of hexose transporters (Leandro et al., 2009): (i) facilitated diffusion carriers, which use an energy-independent mechanism that mediates transport of sugar molecules down their concentration gradient, and (ii) energydependent active proton-sugar symporters, which operate only when sugar concentration is low, and which couple the uptake of sugar molecules to the uptake of protons. The latter uptake system becomes important during growth at low extracellular sugar concentrations.

Hexose transport in S. cerevisiae occurs only via facilitated diffusion carriers and these are encoded by several genes, including the HXT genes (Kruckeberg, 1996; Lagunas, 1993). There are 17 HXT genes in S. cerevisiae, but only seven of these, $H x t 1 p-H x t 7 p$, are required for growth on glucose or fructose (Boles \& Hollenberg, 1997; Luyten et al., 2002; Reifenberger et al., 1997). The proteins encoded by these seven genes have different kinetic properties $\left(K_{\mathrm{m}}\right.$ from 1 to $\left.300 \mathrm{mM}\right)$ and are subject to different modes of regulation. All of the hexose transporters in S. cerevisiae can also transport fructose, but they 
generally have a high $K_{\mathrm{m}}$ for this sugar, indicating glucose to be the preferential sugar for Hxt carriers (Bisson \& Fraenkel, 1983; Reifenberger et al., 1997).

In contrast, Zygosaccharomyces bailii, a fructophilic yeast, has a low-affinity facilitator protein specific for fructose, the Ffzlp transporter (Piña et al., 2004). Fructose may also be transported by other transport systems, such as Fsylp in Saccharomyces pastorianus, the gene of which was the first encoding a specific fructose permease to be identified in yeast. This transporter mediates active fructose uptake by a proton symport mechanism and does not accept glucose as a substrate (Gonçalves et al., 2000). Two other specific fructose $/ \mathrm{H}^{+}$symporters were subsequently characterized in Kluyveromyces lactis (Frtlp) and in the filamentous fungus Botrytis cinerea (Diezemann \& Boles, 2003; Doehlemann et al., 2005). Studies have aimed to establish the repertoire of sugar transporters in yeast, by using functional characterization of proteins or phylogenetic analyses. They have shown that such active fructose $/ \mathrm{H}^{+}$ symporters, although absent in the model yeast $S$. cerevisiae (Rodrigues de Sousa et al., 1995), are actually present in other hemiascomycetous yeasts and in filamentous fungi (Leandro et al., 2009; Palma et al., 2007).

We have recently reported the complete genome sequence of the commercial wine yeast strain EC1118 (Novo et al., 2009). Comparative analysis of this genome with that of the laboratory strain S288C revealed the presence of 34 new genes, grouped into three large clusters located in subtelomeric or internal chromosomal positions. Phylogeny and synteny analyses showed that these gene clusters were acquired by several events of horizontal transfer, two of them (regions A and B) from non-Saccharomyces species and the third (region C) from a species closely related to S. cerevisiae. These genes potentially have key functions in fermentation, such as metabolism of sugars and nitrogen. Among them, 104_6634g from region C, encoded a protein highly similar to that encoded by the $S$. pastorianus-specific fructose symporter FSY1 gene (Novo et al., 2009). The aim of the present study was to characterize the EC-1O4_6634g gene, which we designated FSY1. We report the functional analysis of this gene and demonstrate that it encodes a high-affinity fructose/ $\mathrm{H}^{+}$symporter. In addition, we studied the regulation of this gene during growth on various carbon sources by quantitative RT-PCR. We show that FSY1 expression is repressed by high concentrations of glucose or fructose and is induced by ethanol as the sole carbon source.

\section{METHODS}

Yeast strains and culture conditions. The yeast strains used in this study are listed in Table 1 . Yeast cells were grown at $28{ }^{\circ} \mathrm{C}$ on YP medium ( $1 \%$ Bacto yeast extract, $2 \%$ Bacto peptone) or on synthetic (SD) minimal medium $(0.67 \%$ yeast nitrogen base without amino acids, $50 \mathrm{mg}$ uracil $1^{-1}$ ) containing either $2 \%$ maltose or different amounts of various carbon sources.

For drop-test assays, cells in the exponential phase were pelleted and resuspended in sterile water to an $\mathrm{OD}_{600}$ of 1 . Cells were serially diluted in 10 -fold steps, and $10 \mu \mathrm{l}$ of each dilution was spotted onto a control SD agar plate containing $2 \%(\mathrm{w} / \mathrm{v})$ maltose and onto SD agar plates containing $0.2,2$ or $20 \%(\mathrm{w} / \mathrm{v})$ of either fructose or glucose.

For ${ }^{14} \mathrm{C}$-labelled sugar uptake and symport experiments, cells were cultured in SD minimal medium supplemented with the appropriate carbon source and were subsequently harvested during the midexponential phase of growth $\left(\mathrm{OD}_{600} 0.7-0.9\right)$ by centrifugation and then washed twice with demineralized ice-cold water. Cells were resuspended in citrate buffer and equilibrated at $28{ }^{\circ} \mathrm{C}$ prior to uptake experiments (Spencer-Martins \& Van Uden, 1985).

V5 $h x t 1-7 \Delta$ cells were pre-grown in $2 \%$ maltose, and resuspended to an $\mathrm{OD}_{600}$ of $\sim 0.5$ in fresh medium containing either $0.5 \%(\mathrm{w} / \mathrm{v})$ fructose or $5 \%(\mathrm{w} / \mathrm{v})$ glucose. Cells were then incubated at $28{ }^{\circ} \mathrm{C}$ for $2 \mathrm{~h}$ with shaking and subsequently harvested for sugar transport assays and symport experiments.

Strain construction. The FSY1 gene from strain EC1118 was introduced into strain V5 $h x t 1-7 \Delta$ by genomic integration at the HXT3 locus. A $2.18 \mathrm{~kb}$ DNA fragment was amplified from EC1118 genomic DNA by PCR (cycling conditions: $40 \mathrm{~s}$ at $95{ }^{\circ} \mathrm{C}, 30 \mathrm{~s}$ at $50{ }^{\circ} \mathrm{C}, 5 \mathrm{~min}$ at $68{ }^{\circ} \mathrm{C} ; 25$ cycles) by using the Expand High Fidelity PCR system (Boehringer Mannheim) and the primers 5'-atagaatcacaaacaaaatttacatctgagttaaacaatcatggatctgaataactctgaggatt- $3^{\prime}$, corresponding to the HXT3 promoter (italics) and to nucleotides +1 to +25 of the FSY1 gene (underlined), and aagtgacgggcgatgagtaagaaagaaataactgactcattagatagcctagttttggtggtcatct, corresponding to the HXT7 terminator (italics) and to nucleotides +2078 to +2100 of the FSY1 gene (underlined). The PCR product was used to transform V5 $h x t 1-7 \Delta$ by the lithium acetate method (Gietz \& Woods, 2002). Transformants were selected on SD agar plates containing $50 \mathrm{mg}$ uracil $1^{-1}$ and either a low concentration of fructose $(0.5$ or $1 \%, \mathrm{w} / \mathrm{v})$ or $2 \%(\mathrm{w} / \mathrm{v})$ sucrose.

This construct allowed integration of FSY1 under the control of the HXT3 promoter. Integration of the FSY1 ORF was verified by PCR analysis of total DNA.

Phylogenetic analysis. Protein sequence alignment was built by CLUSTAL $\mathrm{X}$ with homologues retrieved by BLASTP searches against the Fungal Genome and Genolevures databases. Grouping was achieved by using a threshold $E$ value of $<10^{-60}$. For comparison, hexose transporters of $S$. cerevisiae were included. Phylogenetic analysis was done via PhyML v3.0 by using the $\mathrm{WAG}+\mathrm{G}+\mathrm{I}$ model (shape parameter, 2.282; proportion of invariant sites, 0.084) (Guindon \& Gascuel, 2003).

Sugar-uptake and symport experiment. Transport of D$\left[\mathrm{U}_{-}{ }^{14} \mathrm{C}\right]$ glucose and $\mathrm{D}-\left[\mathrm{U}_{-}{ }^{14} \mathrm{C}\right]$ fructose was measured according to the procedures described by Spencer-Martins \& Van Uden (1985). Kinetic parameters were obtained from activity measurements with fructose and glucose concentrations that ranged from 0.05 to $1.4 \mathrm{mM}$ and 0.2 to $80 \mathrm{mM}$, respectively. Eady-Hofstee plots were obtained with Excel software and kinetic parameters were determined by nonlinear regression (Michaelis-Menten equation) by using GraphPad Prism (version 5.02 for Windows, GraphPad Software).

All values reported are expressed as the mean $\pm \mathrm{SD}$ of three independent experiments.

Proton symport activity was studied by using a $\mathrm{pH}$ electrode to record the transient alkalification of an aqueous cell suspension following the addition of sugar, as described by Loureiro-Dias \& Peinado (1984).

Quantitative real-time RT-PCR. Yeast cells were grown at $28{ }^{\circ} \mathrm{C}$ on YP medium with different amounts of various carbon sources to an $\mathrm{OD}_{600}$ of 0.9 or 3 . Total RNA was extracted from $10^{9}$ yeast cells, by using TRIzol reagent (Invitrogen), and was purified on RNeasy spin 
Table 1. S. cerevisiae strains

\begin{tabular}{|c|c|c|}
\hline Strain & Description & Reference/source \\
\hline V5 & MATa ura3 gal & V. Galeote \\
\hline V5 $h x t 1-7 \Delta$ & V5 hxt514:: kanMX hxt367:: kanMX hxt2:: kanMX & Luyten et al. (2002) \\
\hline$V 5$ hxt 1-7D/FSY1 & V5 hxt514:: kanMX hxt367:: FSY1 hxt2:: kanMX & This study \\
\hline Lalvin EC1118 & Champagne wine strain & Lallemand Inc. \\
\hline
\end{tabular}

columns (Qiagen). Purified RNA was quantified with a spectrophotometer (Nanodrop 1000; Thermoscientific) and its integrity was assessed by electrophoresis. Purified RNA was treated with DNase I (Invitrogen) and first-strand cDNA synthesis was carried out by using SuperScript III reverse transcriptase (Invitrogen) and random primers, in accordance with the manufacturer's protocol. Quantitative PCRs were performed in triplicate on an ABI 7300 real-time PCR system (Applied Biosystems), using Power SYBR Green PCR Master Mix (Applied Biosystems) and gene-specific primers. The SCR1 gene was amplified as an internal control. The DNA sequences of the primers were as follows: FSY1, gctttgctaatcggcaccat (F) and aagcacgtctgccaaatctgt (R); SCR1, tctggtgcggcaaggtagtt (F) and cacctttgctgacgctggat $(\mathrm{R})$.

The comparative $C_{\mathrm{t}}$ method was used for quantification of gene expression (Livak \& Schmittgen, 2001). Gene expression was normalized with respect to the expression of SCR1, a non-coding RNA gene with a constant level of expression (data not shown). Normalized expression levels were compared with the lowest level of expression of FSY1, which occurred at a concentration of $2 \%$ glucose at an $\mathrm{OD}_{600}$ of 0.9 .

\section{RESULTS}

\section{Phylogenetic analysis of the FSY1 gene}

Analysis of the genome sequence of strain EC1118 revealed 34 new genes that were not present in the S288C laboratory strain (Novo et al., 2009). A search of the UniProt-KB, GenBank and Genolevures databases identified homologous proteins. Of these, the $S$. pastorianus fructose-specific symporter Fsylp had $78 \%$ amino-acid similarity with the protein encoded by strain EC1118 (104_6634g). This gene, designated FSY1, consists of a 1701 bp ORF encoding a predicted protein of 567 amino acids.

Phylogenetic analysis showed a significant level of similarity with other yeast high-affinity fructose transporters (Fig. 1). We observed two distinct clusters, one including three functionally characterized high-affinity fructose symporters (Fsylp from S. pastorianus and the two Frtlp proteins from $K$. lactis and $B$. cinerea) and several non-characterized sequences, and the other including the hexose facilitator proteins. The highest level of similarity was with $S$. pastorianus and Saccharomyces uvarum transporters (78\% amino acid similarity), while that with $K$. lactis and $B$. cinerea Frt1p was lower (66 and $44 \%$, respectively).

\section{Functional analysis of the FSY1 gene}

To examine the functionality of the FSY1 gene, we expressed FSY1 under the control of the HXT3 promoter, in strain V5 hxt1-7A. This strain lacks the hexose transporter genes HXT1 to HXT7 and cannot utilize glucose, fructose or sucrose as sole carbon source (Luyten et al., 2002). As $S$. pastorianus Fsylp has a low $K_{\mathrm{m}}$ for fructose (Gonçalves et al., 2000), transformants were selected on minimal medium containing uracil and either a low concentration of fructose $(0.5$ or $1 \%)$ or $2 \%$ sucrose (which was converted into small amounts of glucose and fructose by invertase). Strains with the integrated FSY1 gene were obtained on all these media. The FSY1 gene was therefore able to complement the growth deficiency of the hxt1-7D mutant, indicating that it encodes a hexose transporter.

We examined the growth of one of these strains in comparison with V5 and V5 $h x t 1-7 \Delta$ by using drop-test assays on plates containing minimal medium with different concentrations of either glucose or fructose. As expected, V5 $h x t 1-7 \Delta$ could not grow on glucose or fructose as sole carbon source, whereas all the strains grew well on maltose, which is taken up by a specific transport system (Fig. 2). FSY1 expression was able to complement the growth

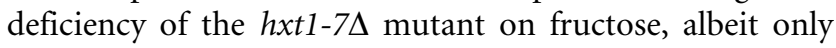
partially on $20 \%$ fructose medium (Fig. 2b). Similar properties have been reported for the $S$. pastorianus and $K$. lactis high-affinity fructose $/ \mathrm{H}^{+}$symporters (Diezemann \& Boles, 2003; Gonçalves et al., 2000).

Expression of the FSY1 gene also partially restored growth of V $5 x t 1-7 \Delta$ on glucose, although to a considerably lesser extent than on fructose (Fig. 2b). Growth of V5, V5 hxt1$7 \Delta$ and V5 $h x t 1-7 \Delta / F S Y 1$ was also monitored in liquid SD medium supplemented with uracil and containing different carbon sources (Fig. 3). FSY1 restored the growth of V5 $h x t 1-7 \Delta$ in 0.5 and $5 \%$ fructose and in $5 \%$ glucose, thus confirming the result of the drop test assays.

\section{Symport activity}

Fructose $/ \mathrm{H}^{+}$symport activity was measured by alkalinization of cell suspensions after the addition of sugars. Proton influx resulting in external alkalinization was observed when fructose was added at a final concentration of $5 \mathrm{mM}$ to aqueous suspensions of cells expressing FSY1 that were pre-grown in fructose medium (Fig. 4a), as indicated by the increase in alkalinization rate triggered by addition of fructose. Activation of the plasma membrane ATPase resulting from fructose metabolism was also subsequently observed, as expected. Fructose failed to elicit changes in the net rate of proton movements for V5 $h x t 1-7 \Delta$, strongly 


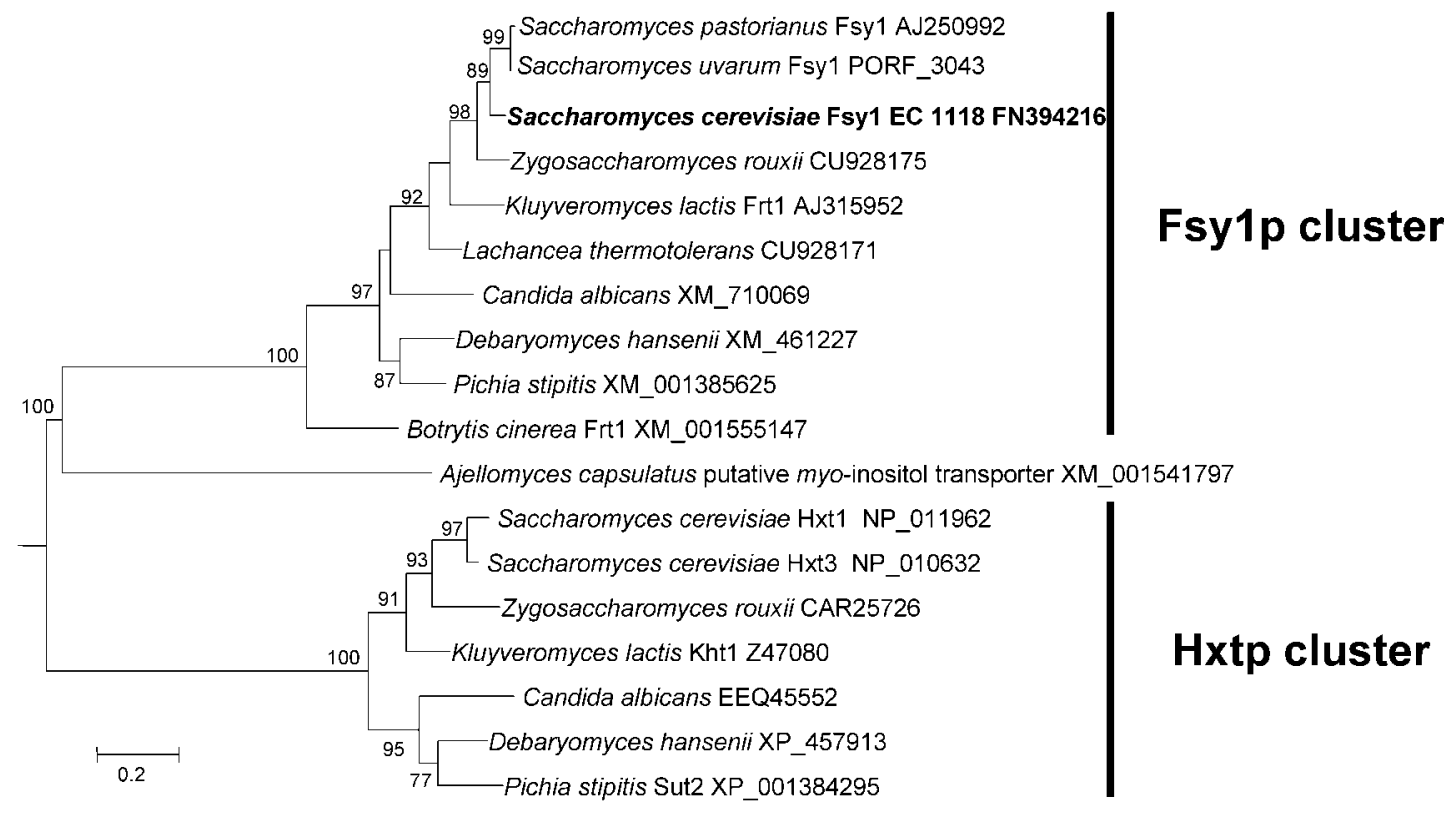

Fig. 1. Phylogenetic relationship between the $S$. cerevisiae Fsy EC1118 protein (in bold) and other fungal transporters, showing the Hxt and Fsy1 clusters. GenBank accession numbers or ORF codes are indicated after the species designation. Protein acronyms are only noted for transporters that have been biochemically characterized. The Ajellomyces capsulatus putative myo-inositol transporter is the closest relative of the Fsy1 cluster proteins, as judged by BLAST searches. Numbers at nodes are bootstrap values based on 1000 replicates; only values $>50 \%$ are shown.

suggesting that this strain is unable to transport or metabolize fructose (Fig. 4a). These results demonstrate that FSY1, like the FSY1 gene of S. pastorianus and the FRT1 gene, encodes a proton-coupled fructose transporter. Addition of L-sorbose to aqueous suspensions of cells also produced proton influx but because this sugar is not metabolized we did not observe a subsequent decrease in
$\mathrm{pH}$ (data not shown). Similar results have been obtained with the FSY1 gene of S. pastorianus (Cason et al., 1986).

Weak acidification was observed when V5 $h x t 1-7 \Delta / F S Y 1$ cells were grown on glucose (Fig. 4b), consistent with the partial restoration of growth observed on glucose plates or liquid medium. However, alkalinization was not observed, (a)

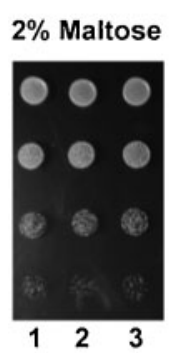

(b)
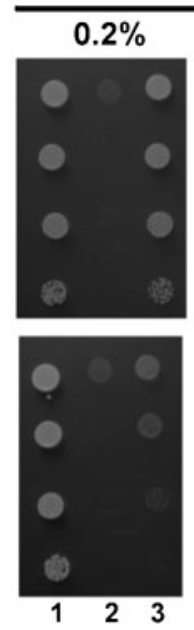

\section{Sugar $(\%, w / v)$}
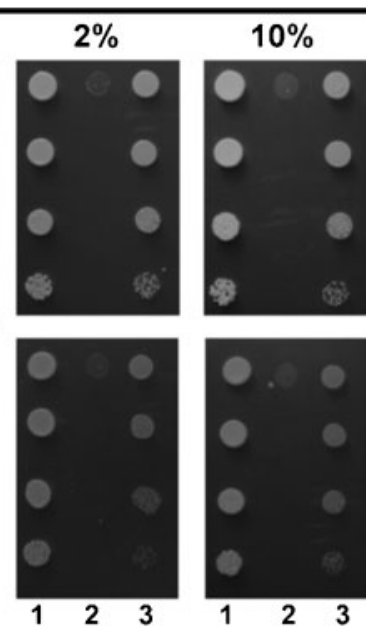
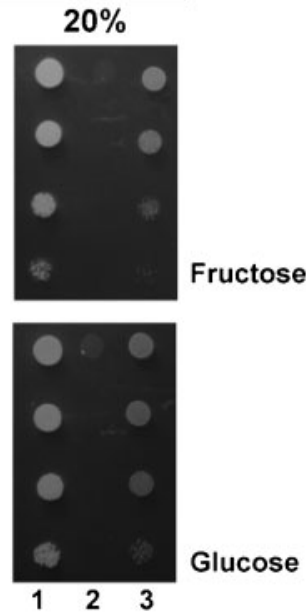

Fig. 2. Functional analysis of the FSY1 fructose transporter expressed in $S$. cerevisiae $h x t 1-7 \Delta$ strain. The V5 strain (1) served as a wild-type control. FSY1 under control of the HXT3 promoter (3) was integrated into the isogenic $h x t 1-7 \Delta$ mutant. The V5 $h x t 1-7 \Delta$ strain (2) was used as a negative control. The different yeast strains were grown for 2 days on solid minimal medium supplemented with $2 \%$ maltose (a) or different concentrations of fructose or glucose (b). 


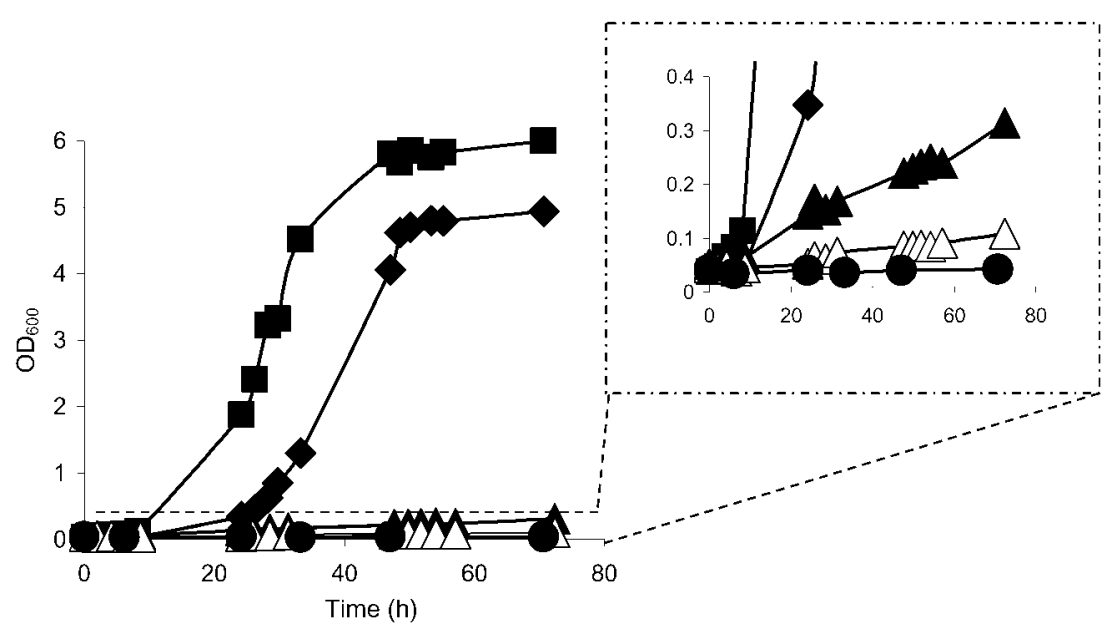

Fig. 3. Growth of $V 5 h x t 1-7 \Delta$ expressing the $F S Y 1$ gene on synthetic medium with different carbon sources. Growth was monitored by measuring $\mathrm{OD}_{600}$ at the times indicated. $\boldsymbol{\square}, 5 \%$ fructose;, $0.5 \%$ fructose; $\boldsymbol{\Delta}, 5 \%$ glucose; $\triangle, 0.5 \%$ glucose;

- V5 $h x t 1-7 \Delta$ on $5 \%$ fructose. A zoom of the $\mathrm{OD}_{600}$ area from 0 to 0.4 is also shown.

possibly because the transport capacity for glucose is very low (see next section).

\section{Kinetic parameters of hexose transport}

Sugar uptake was measured in V5 $h x t 1-7 \Delta / F S Y 1$ cells grown on synthetic medium containing $0.5 \%$ fructose. As a control, sugar uptake was measured in V5 $h x t 1-7 \Delta$ cells
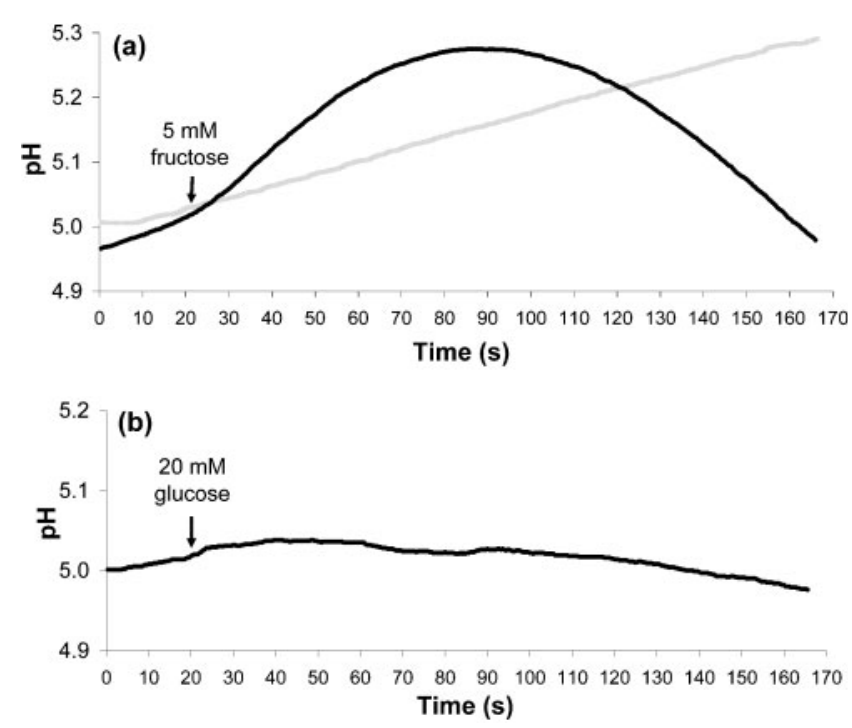

Fig. 4. Energy-coupled transport by Fsy1p. Effects of the addition of sugars on the $\mathrm{pH}$ of an aqueous suspension of (a) $\mathrm{V} 5 h \times t 1-7 \Delta$ cells pre-grown on maltose and incubated for $2 \mathrm{~h}$ in fructose medium (grey line) and V5 $h x t 1-7 \Delta / F S Y 1$ grown on fructose (dark line), and (b) V5 $h x t 1-7 \Delta / F S Y 1$ cells grown on $5 \%$ glucose. that were grown on the same medium containing $2 \%$ maltose and then incubated in $0.5 \%$ fructose for $2 \mathrm{~h}$.

The kinetic parameters of fructose uptake mediated by FSY1 were determined in strain V5 $h x t 1-7 \Delta$ expressing FSY1. $K_{\mathrm{m}}$ values of $0.24 \pm 0.04 \mathrm{mM}$ with a $V_{\max }$ of $0.93 \pm$ $0.08 \mathrm{mmol} \mathrm{h}^{-1}$ (g dry weight $)^{-1}$ were obtained. No uptake of fructose could be measured in strain V5 hxt1-7D, indicating that the transport kinetic constants determined with the V5 hxt1-7D/FSY1 strain represent the kinetic properties of the S. cerevsiae EC1118 Fsylp carrier. Although the $K_{\mathrm{m}}$ value was somewhat higher for $F S Y 1$, it is consistent with the values previously reported $(0.16 \pm 0.02 \mathrm{mM})$ for Fsylp of $S$. pastorianus and for Frtlp of K. lactis (Diezemann \& Boles, 2003; Gonçalves et al., 2000). We also tried to measure glucose transport (D-[U- $\left.{ }^{14} \mathrm{C}\right]$ glucose concentration between 0.2 and $80 \mathrm{mM}$ ) in cells pre-grown under different conditions, but no significant glucose uptake was detected (results not shown). Furthermore, in competition experiments, realized by addition of $125 \mathrm{mM}$ unlabelled glucose to $\mathrm{D}-\left[\mathrm{U}-{ }^{14} \mathrm{C}\right]$ fructose (concentration range $0.05-1.4 \mathrm{mM}$ ), glucose did not inhibit $\left[{ }^{14} \mathrm{C}\right]$ fructose uptake by yeast cells expressing FSY1; indeed, the same $K_{\mathrm{m}}$ and $V_{\max }$ values were obtained. This may be due to the extremely low affinity of this transporter for glucose.

\section{Expression of FSY1 in EC1118}

Expression of the FSY1 gene in strain EC1118 was analysed in various growth conditions by quantitative real-time RTPCR. Total RNA was purified from strain EC1118 grown with either 0.5 or $2 \%$ fructose or glucose, $2 \%$ ethanol or $2 \%$ maltose, at two different growth stages $\left(\mathrm{OD}_{600} 0.9\right.$ and 3). Transcript concentrations of FSY1 are shown in Fig. 5(a). A lower transcript abundance was observed in glucose- or fructose-grown cells collected at an $\mathrm{OD}_{600}$ of 

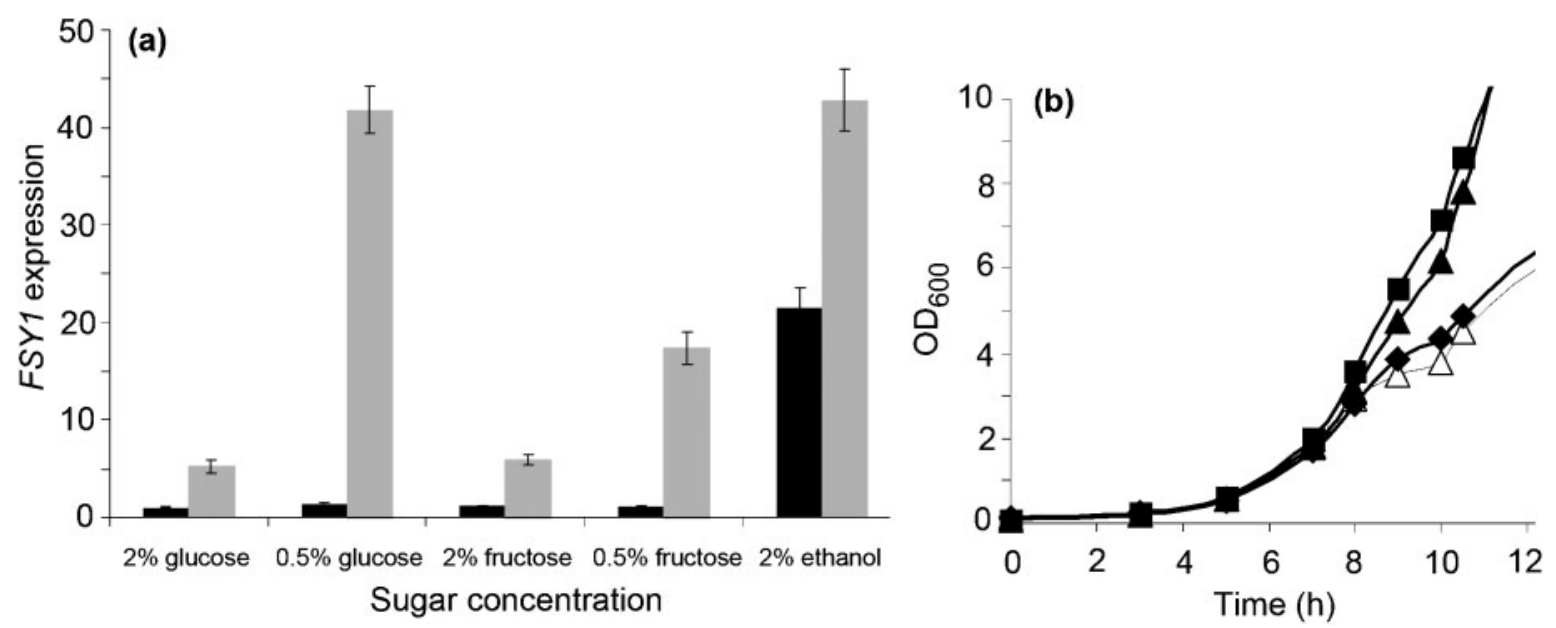

Fig. 5. Quantitative RT-PCR of FSY1 gene expression in strain EC1118 grown in YP medium containing different amounts of various carbon sources at $28{ }^{\circ} \mathrm{C}$. All media were inoculated (initial $\mathrm{OD}_{600} 0.1$ ) with cells pre-grown on YP plus $2 \%$ maltose. (a) Cells were collected at $\mathrm{OD}_{600} 0.9 \pm 0.04$ (black bars) or $\mathrm{OD}_{600} 3 \pm 0.08$ (grey bars). RNA was extracted and analysed by quantitative RT-PCR. The relative expression of $F S Y 1$ was normalized to its expression in $2 \%$ glucose at $\mathrm{OD}_{600} 0.9$. The results are expressed as the mean \pm SD of relative expression from three repeat experiments. (b) Growth on hexoses was monitored by measuring the $\mathrm{OD}_{600}$ at the times indicated. $\boldsymbol{\Lambda}, 2 \%$ glucose; $\triangle, 0.5 \%$ glucose; $\mathbf{\square}, 2 \%$ fructose;, $0.5 \%$ fructose.

0.9 whereas the gene was highly expressed on ethanol at this growth stage. Transcript levels of FSY1 increased with depletion of hexoses from the growth medium. This increase was particularly noticeable for cells grown on $0.5 \%$ glucose or fructose at an $\mathrm{OD}_{600}$ of 3 (at $8 \mathrm{~h}$ ), when most of the sugar was exhausted, as suggested by the observation of a diauxic shift shortly after the sampling time point (at around $9 \mathrm{~h}$ ) (Fig. 5b). The difference in transcript abundance in these two conditions was probably related to a difference in the residual sugar concentration.

These data indicate that expression of FSY1 is repressed by high glucose or fructose concentrations and is up-regulated on non-fermentable carbon sources. A higher transcript concentration of FSY1 was observed on maltose than on glucose, consistent with maltose being less effective in catabolite repression than glucose (Eraso \& Gancedo, 1984).

\section{DISCUSSION}

The present study describes the functional characterization of an S. cerevisiae-specific fructose transporter, Fsylp, discovered by genome sequencing of the wine yeast strain EC1118 and also found in many other wine yeast $S$. cerevisiae strains (Novo et al., 2009). So far, only hexose uniporters have been identified in S. cerevisiae, which makes Fsylp the first active fructose transporter carrier to be described in this species.

Novo et al. (2009) have analysed the distribution of the EC1118 genomic region containing the FSY1 gene among $S$. cerevisiae clades. They propose that this $65 \mathrm{~kb}$ region was obtained by horizontal gene transfer, probably from a species closely related to the genus Saccharomyces. No PCR amplification was obtained with primers based on the sequence of the FSY1 gene with the strains dispersed among the 'Saccharomyces complex' described by Kurtzman \& Robnett (2003) (data not shown). However, phylogenetic analysis of Fsylp showed that the two closest homologues were the functionally characterized fructose $/ \mathrm{H}^{+}$symporter Fsylp of $S$. pastorianus, and the structurally related fructose transporter of S. uvarum ( $78 \%$ similarity). These findings are consistent with the hypothesis that the contributor of this region may be an as yet undescribed species very closely related to the genus Saccharomyces (Novo et al., 2009).

Functional complementation experiments in V5 $h x t 1-7 \Delta$, a mutant strain unable to ferment hexoses, and kinetic characterization revealed that Fsylp of $S$. cerevisiae is a high-affinity fructose $/ \mathrm{H}^{+}$symporter with characteristics similar to those of S. pastorianus Fsylp. The low growth observed in glucose medium and the weak acidification shown in symport experiments led to the assumption that this protein can also transport glucose. However, no uptake of glucose could be measured and no inhibition of fructose transport by glucose was observed. This might be explained by a very low affinity of this transporter for glucose, in line with the slightly improved growth of the V5 $h x t 1-7 \Delta / F S Y 1$ strain at high glucose concentrations. To identify small differences between the kinetic properties of the different Fsylp homologues, it will be necessary to express all the proteins from the same promoter in the same strain background.

Expression of S. cerevisiae FSY1 is regulated by the carbon source and was maximal during respiratory growth on 
ethanol. Although the transcriptional regulation of FSY1 is clearly repressed by glucose or fructose, this gene was expressed at detectable levels whatever the carbon source and the amount of sugar. A different regulation has been observed for the FRT1 gene of $K$. lactis, which had no detectable expression in ethanol-grown cells and was not subject to sugar catabolic repression (Diezemann \& Boles, 2003).

Compared with its close relative S. pastorianus, Fsylp seems to be able to transport glucose, albeit to a much lesser extent. On the other hand, phylogenetically more distant transporters are not repressed by glucose (K. lactis) and were able to rescue growth of an hxt-null mutant in $2 \%$ glucose medium (Diezemann \& Boles, 2003; Doehlemann et al., 2005). In a recent review on the evolution of hexose transport across Ascomycota fungi, Leandro et al. (2009) proposed that the FSY1 gene appeared early during evolution of ascomycetes and has subsequently been selectively lost in some lineages of both yeasts and moulds. In hemiascomycetes, putative orthologues of FSY1 are well conserved through the CTG clade (a monophyletic clade within the Saccharomycotina containing organisms that translate CTG as serine instead of leucine) (Fitzpatrick et al., 2006) and Kluyveromyces clade. Indeed, in addition to the putative orthologues shown in Fig. 1, five other species among these two clades have homologues to Fsylp (Lanchancea kluyveri, Pichia guilliermondii, Candida tropicalis, Candida dubliniensis, Lodderomyces elongisporus) (data not shown). This phylogenetic tree combined with the differential regulation of the Saccharomyces and K. lactis fructose transporter genes suggest that the fructose transporter has undergone a clear functional divergence in the evolution of these different lineages. In light of these various studies, we can hypothesize that among the 'Saccharomyces complex', fructose $/ \mathrm{H}^{+}$symporters have been lost or have evolved a fine-tuned regulation that led to the emergence of a new function. Subsequently, some $S$. cerevisiae oenological strains might have gained an evolved gene by horizontal gene transfer.

The discrepancy in glucose and fructose utilization by $S$. cerevisiae is an essential step during oenological fermentation. The role and importance of sugar transport in oenological conditions were emphasized by Luyten et al. (2002), who showed that HXT1, HXT2, HXT3, HXT6 and HXT7 are required for optimal sugar fermentation in grape must. On the other hand, Guillaume et al. (2007) showed that the higher fructose utilization capacity of the Fermichamp wine yeast strain results from the expression of an allelic variant of HXT3. As fructose utilization is rate limiting at the end of fermentation, this allele has probably been retained in some wine yeasts because it confers a selective advantage during wine fermentation.

Similarly, FSY1 might have been selected and retained in wine yeasts because of the selective advantage it may confer. Most of the new genes acquired by strain EC1118 are present in the Champagne group containing EC1118-related strains, and in a closely related group containing flor yeast (Novo et al., 2009). S. cerevisiae flor yeasts form a film (or velum) on the surface of the wine, and develop an oxidative metabolism in the presence of a high ethanol concentration and low levels of fermentable sugar, essentially fructose (Berlanga et al., 2001; Charpentier et al., 2009). In light of the characteristics of the Fsylp of strain EC1118, the acquisition of FSY1 by horizontal transfer could confer an advantage either at the end of wine fermentation, when wine yeasts have to ferment this non-preferred sugar in the presence of large amounts of ethanol, or in the oxidative flor conditions.

Overall, these data highlight the potential role of environmental pressure on the acquisition of new functions by yeast. Fructose transport capacity and resistance to ethanol are key parameters for improving the fermentation performance of wine yeasts (Guillaume et al., 2007; Santos et al., 2008). Future studies on the role of Fsylp in various wine-making situations and on its sensitivity to ethanol should provide further support to the hypothesis that the acquisition of this transporter by $S$. cerevisiae has an adaptive value in the wine environment.

\section{ACKNOWLEDGEMENTS}

The authors thank Frédéric Bigey, Jean-Luc Legras and Bruno Blondin for helpful discussions and Brigitte Cambon for technical assistance. E. V. was supported by the Fundação para a Ciência e a Tecnologia, Portugal (grant SFRH/BPD/46471/2008).

\section{REFERENCES}

Berlanga, T. M., Atanasio, C., Mauricio, J. C. \& Ortega, J. M. (2001). Influence of aeration on the physiological activity of flor yeasts. J Agric Food Chem 49, 3378-3384.

Berthels, N. J., Cordero Otero, R. R., Bauer, F. F., Thevelein, J. M. \& Pretorius, I. S. (2004). Discrepancy in glucose and fructose utilisation during fermentation by Saccharomyces cerevisiae wine yeast strains. FEMS Yeast Res 4, 683-689.

Bisson, L. F. (1999). Stuck and sluggish fermentations. Am J Enol Vitic 50, 107-119.

Bisson, L. F. \& Fraenkel, D. G. (1983). Involvement of kinases in glucose and fructose uptake by Saccharomyces cerevisiae. Proc Natl Acad Sci U S A 80, 1730-1734.

Boles, E. \& Hollenberg, C. P. (1997). The molecular genetics of hexose transport in yeasts. FEMS Microbiol Rev 21, 85-111.

Cason, D. T., Spencer-Martins, I. \& van Uden, N. (1986). Transport of fructose by a proton symport in a brewing yeast. FEMS Microbiol Lett 36, 307-309.

Charpentier, C., Colin, A., Alais, A. \& Legras, J. L. (2009). French Jura flor yeasts: genotype and technological diversity. Antonie van Leeuwenhoek 95, 263-273.

Diezemann, A. \& Boles, E. (2003). Functional characterization of the Frt1 sugar transporter and of fructose uptake in Kluyveromyces lactis. Curr Genet 43, 281-288.

Doehlemann, G., Molitor, F. \& Hahn, M. (2005). Molecular and functional characterization of a fructose specific transporter from the gray mold fungus Botrytis cinerea. Fungal Genet Biol 42, 601-610.

Eraso, P. \& Gancedo, J. M. (1984). Catabolite repression in yeasts is not associated with low levels of cAMP. Eur J Biochem 141, 195-198. 
Fitzpatrick, D. A., Logue, M., Stajich, J. \& Butler, G. (2006). A fungal phylogeny based on 42 complete genomes derived from supertree and combined gene analysis. BMC Evol Biol 6, 99.

Gafner, J. \& Schütz, M. (1996). Impact of glucose-fructose ratio on stuck fermentations: practical experience to restart stuck fermentations. Vitic Enol Sci 51, 214-218.

Gietz, R. D. \& Woods, R. A. (2002). Transformation of yeast by lithium acetate single-stranded carrier DNA polyethylene glycol method. In Methods in Enzymology: Guide to Yeast Genetics and Molecular and Cell Biology, pp. 87-96. Edited by G. R. Fink \& C. Gutherie. New York: Academic Press.

Gonçalves, P., Rodrigues de Sousa, H. \& Spencer-Martins, I. (2000). FSY1, a novel gene encoding a specific fructose $/ \mathrm{H}^{+}$symporter in the type strain of Saccharomyces carlsbergensis. J Bacteriol 182, 5628-5630.

Guillaume, C., Delobel, P., Sablayrolles, J. M. \& Blondin, B. (2007). Molecular basis of fructose utilization by the wine yeast Saccharomyces cerevisiae: a mutated HXT3 allele enhances fructose fermentation. Appl Environ Microbiol 73, 2432-2439.

Guindon, S. \& Gascuel, O. (2003). A simple, fast, and accurate algorithm to estimate large phylogenies by maximum likelihood. Syst Biol 52, 696-704.

Kruckeberg, A. L. (1996). The hexose transporter family of Saccharomyces cerevisiae. Arch Microbiol 166, 283-292.

Kurtzman, C. P. \& Robnett, C. J. (2003). Phylogenetic relationships among yeasts of the 'Saccharomyces complex' determined from multigene sequence analyses. FEMS Yeast Res 3, 417-432.

Lagunas, R. (1993). Sugar transport in Saccharomyces cerevisiae. FEMS Microbiol Rev 10, 229-242.

Leandro, M. J., Fonseca, C. \& Gonçalves, P. (2009). Hexose and pentose transport in ascomycetous yeasts: an overview. FEMS Yeast Res 9, 511-525.

Livak, K. J. \& Schmittgen, T. D. (2001). Analysis of relative gene expression data using real-time quantitative PCR and the $2^{-\Delta \Delta C_{1}}$ method. Methods 25, 402-408.
Loureiro-Dias, M. C. \& Peinado, J. M. (1984). Transport of maltose in Saccharomyces cerevisiae. Effect of $\mathrm{pH}$ and potassium ions. Biochem $\mathrm{J}$ 222, 293-298.

Luyten, K., Riou, C. \& Blondin, B. (2002). The hexose transporters of Saccharomyces cerevisiae play different roles during enological fermentation. Yeast 19, 713-726.

Novo, M., Bigey, F., Beyne, E., Galeote, V., Gavory, F., Mallet, S., Cambon, B., Legras, J. L., Wincker, P. \& other authors (2009). Eukaryote-to-eukaryote gene transfer events revealed by the genome sequence of the wine yeast Saccharomyces cerevisiae EC1118. Proc Natl Acad Sci U S A 106, 16333-16338.

Palma, M., Goffeau, A., Spencer-Martins, I. \& Baret, P. V. (2007). A phylogenetic analysis of the sugar porters in hemiascomycetous yeasts. J Mol Microbiol Biotechnol 12, 241-248.

Piña, C., Gonçalves, P., Prista, C. \& Loureiro-Dias, M. C. (2004). Ffz1, a new transporter specific for fructose from Zygosaccharomyces bailii. Microbiology 150, 2429-2433.

Reifenberger, E., Boles, E. \& Ciriacy, M. (1997). Kinetic characterization of individual hexose transporters of Saccharomyces cerevisiae and their relation to the triggering mechanisms of glucose repression. Eur J Biochem 245, 324-333.

Rodrigues de Sousa, H., Madeira-Lopes, A. \& Spencer-Martins, I. (1995). The significance of active fructose transport and maximum temperature for growth in the taxonomy of Saccharomyces sensu stricto. Syst Appl Microbiol 18, 44-51.

Santos, J., Sousa, M. J., Cardoso, H., Inácio, J., Silva, S., SpencerMartins, I. \& Leão, C. (2008). Ethanol tolerance of sugar transport, and the rectification of stuck wine fermentations. Microbiology 154, $422-430$.

Spencer-Martins, I. \& Van Uden, N. (1985). Catabolite interconversion of glucose transport systems in the yeast Candida wickerhamii. Biochim Biophys Acta 812, 168-172.

Edited by: K. Haynes 